\title{
ESPECIAÇÃO DE METAIS-TRAÇO EM SEDIMENTOS DE FLORESTAS DE MANGUEZAIS COM AVICENNIA E RHIZOPHORA
}

Regina Célia Bastos de Andrade e Sambasiva Rao Patchineelam

Departamento de Geoquímica Ambiental, Instituto de Química - Universidade Federal Fluminense - 24020-007 - Niterói - RJ

Recebido em 25/8/99; aceito em 17/5/00

\begin{abstract}
METAL SPECIATION UNDER RHIZOPHORA AND AVICENNIA MANGLES. Speciation studies of $\mathrm{Fe}, \mathrm{Cr}, \mathrm{Co}, \mathrm{Ni}$ and $\mathrm{Cu}$ on reactive fraction (adsorved on oxides, hydroxides, carbonates and clay minerals) and pyrite were performed in Avicennia schaueriana and Rhizophora mangle sediments from Amapá shoreline-Brazil. The soil under Avicennia showed a higher heavy metal concentration in reactive fraction than under Rhizophora. The soil under Rhizophora showed low heavy metal bioavailability, having an increasing association with pyrite across sediment section.
\end{abstract}

Keywords: trace metals; mangroves; speciation.

\section{INTRODUÇÃO}

O sedimento de mangue com a espécie Rhizophora mangle mostra uma maior concentração de compostos de enxofre reduzido, que aqueles recobertos com Avicennia ${ }^{21}$. A rizosfera da Avicennia tende a ser mais rica em matéria orgânica e possui um $\mathrm{pH}$ mais elevado7,14. A Rhizophora influencia de forma menos efetiva as condições gerais do solo abaixo de sua rizosfera, sendo por conseguinte altamente sulfídrica ${ }^{7,10,21}$.

Diversos estudos têm demonstrado as interações entre os metais-traço e os minerais de sulfetos de ferro autigênicos em ambiente sedimentar anóxico ${ }^{11,13}$. O comportamento destes metais está diretamente associado às condições físico-químicas no sedimento e água intersticial ${ }^{4,12}$

Este trabalho tem como objetivo estudar a distribuição de $\mathrm{Fe}, \mathrm{Co}, \mathrm{Cr}$, Ni e $\mathrm{Cu}$ associados as frações reativa e pirita em sedimento recoberto por diferentes espécies de mangue em ambiente sem influência antropogênica.

\section{ÁREA DE ESTUDO}

O presente estudo foi feito na costa norte Amapense, uma região sem impactação, onde há o predomínio dos processos biogeoquímicos naturais. O litoral do Amapá recebe aproximadamente $1.2 \times 10^{8}$ ton/ano de sedimento da bacia de drenagem amazônica, formando extensos depósitos de sedimento fino ${ }^{1}$. Mendes e Faria ${ }^{9}$ mostraram uma abordagem geológica detalhada da área citada .

Amostras de sedimento foram coletadas com perfiladores de 4 - 25 de março/1996, durante o cruzeiro feito pelo navio R/V Seward Johnson, em duas estações com franja de mangue. A primeira em sedimento recoberto por Rhizophora - Cabo Cassiporé $\left(03^{\circ} 54.700 \mathrm{~N} 51^{\circ} 06.375 \mathrm{~W}\right.$ - estação 1) e a segunda com Avicennia - Ponta da Costa $\left(04^{\circ} 06.405 \mathrm{~N} 51^{\circ} 10.600 \mathrm{~W}\right.$ estação 2) (Figura 1).

\section{Amostragem e Metodologia:}

Foram coletados dois testemunhos de $30 \mathrm{~cm}$ para ambas as estações e um de $15 \mathrm{~cm}$ para a estação 2 . Os testemunhos foram seccionados em atmosfera inerte (disposição das secções nas Tabelas 3-6), e mantidos em refrigeração. Para garantir a

e-mails: regina@geoq.uff.br; geosam@vm.uff.br

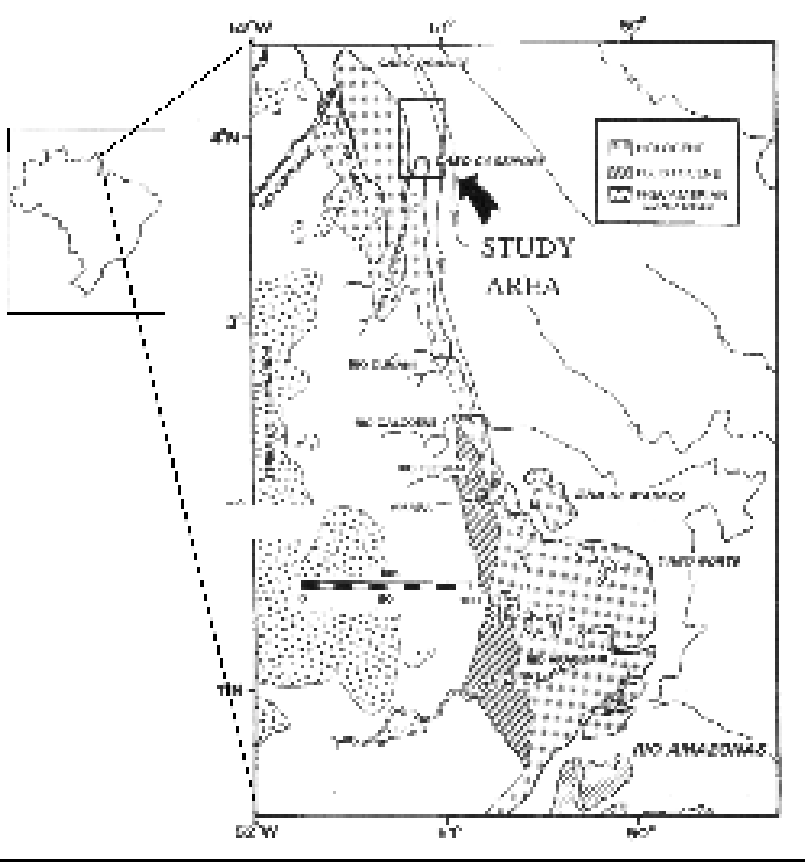

Figura 1. Mapa geológico do Amapá.

'integridade das amostras', as mesmas foram liofilizadas e mantidas em dessecador até análise. Esta diferenciação na coleta dos testemunhos foi feita com o intuito de estudar diferentes processos biogeoquímicos ocorridos ao longo da coluna sedimentar nos pontos amostrados; a estação 2 em função da espécie predominante, seria um ambiente deposicional mais estável que o ponto 1 no Cabo Cassiporé.

Em cada fração seccionada aplicou-se o método de extração seqüencial e seletiva para metais traço associados a fração pirita ${ }^{5}$. Este método extrai seqüencialmente quatro frações: 1) Reativa - obtida após digestão por $16 \mathrm{~h}$. com $\mathrm{HCl}$ $1 \mathrm{M}$, compreendendo metais associados a oxi-hidróxidos amorfos ou cristalinos, carbonatos e argilo-minerais, bem como adsorvidos a sulfetos metaestáveis 2) Silicatos - duas lixiviações consecutivas com HF $10 \mathrm{M}$ por 1 e $16 \mathrm{~h}$, seguidas da redissolução dos fluoretos metálicos formados com ácido bórico; esta fração compreende os metais na rede cristalina 3) Matéria Orgânica - digestão com $\mathrm{H}_{2} \mathrm{SO}_{4}$ conc. por $2 \mathrm{~h}$., 
Tabela 1. Abertura de amostras em forno microondas para obtenção da concentração total.

\begin{tabular}{cccccc}
\hline \multicolumn{5}{c}{ ABERTURA DE AMOSTRAS UTILIZANDO O MICROONDAS } \\
\hline Fe & $\mathrm{Co}$ & $\mathrm{Ni}$ & $\mathrm{Cu}$ & $\mathrm{Cr}$ \\
Padrão & 3,77 & 9,30 & 27,00 & 24,00 & 70,70 \\
Padrão Duplicata & 2,89 & 10,00 & 32,00 & 30,00 & 79,90 \\
Média & $3,33 \pm 0,62$ & $9,65 \pm 0.50$ & $29,50 \pm 3.54$ & $27,00 \pm 4,24$ & $75,30 \pm 6,50$ \\
Valor Verdadeiro & 3,35 & 10,50 & 32,00 & 18,00 & 76,00 \\
Amostra "X" & 4,21 & 14,00 & 30,00 & 49,00 & 69,00 \\
Amostra "X" Duplicata & 4,35 & 14,00 & 29,00 & 40,60 & 60,00 \\
Média & $4,28 \pm 0,10$ & $14,00 \pm 0,00$ & $29,50 \pm 0,70$ & $44,80 \pm 5,94$ & $64,50 \pm 6,36$ \\
\hline
\end{tabular}

*Fe em \% e $\mathrm{Co}, \mathrm{Ni}, \mathrm{Cu}$ e $\mathrm{Cr}$ em $\mathrm{mg} / \mathrm{kg}$.

Tabela 2. Utilização da extração sequencial (HUERTA-DIAZ \& MORSE, 1990) para obtenção da concentração total (somatório das frações reativa, silicato, matéria orgânica e pirita).

\begin{tabular}{cccccc}
\hline \multicolumn{5}{c}{ Extração Sequencial } \\
\hline & $\mathrm{Fe}$ & $\mathrm{Co}$ & $\mathrm{Ni}$ & $\mathrm{Cu}$ & $\mathrm{Cr}$ \\
Amostra "X" & 4,74 & 15,80 & 26,80 & 32,00 & 54,00 \\
Amostra "X" Duplicata & 4,30 & 13,40 & 23,40 & 44,60 & 64,00 \\
Média & $4,52 \pm 0,31$ & $14,60 \pm 1,70$ & $25,10 \pm 2,40$ & $38,30 \pm 8,90$ & $59,00 \pm 7,07$ \\
\hline
\end{tabular}

*Fe em \% e Co, $\mathrm{Ni}, \mathrm{Cu}$ e $\mathrm{Cr}$ em $\mathrm{mg} / \mathrm{kg}$.

obtendo-se os metais associados ao material orgânico 4) Pirita - digestão com $\mathrm{HNO}_{3}$ conc. por 2h., compreendendo os metais associados à pirita.

A exatidão das análises foi testada através da abertura total em microondas de padrão internacional 'NIST' (Nacional Institute of Standards and Technology, Estuarine Sediment-1646) e de amostra " $X$ ", selecionada aleatoriamente dentre as amostras seccionadas; as concentrações totais da amostra "X" para os metais estudados foram comparadas às concentrações totais obtidas pela extração seqüencial ( totalizando-se as quatro frações para cada metal pelo método otimizado ${ }^{5}$ ). As amostras foram lidas em ICP-MS (Espectrometria de Massa com fonte de Plasma Indutivamente Acoplado) no CENPES-PETROBRAS, com coeficiente de correlação para os metais superior a 0.999 e precisão das leituras do aparelho de $3 \%$. Todos os reagentes utilizados possuíam alto grau de pureza. A água usada foi do tipo Milli-Q, passada em Ultrassom. Os resultados do ajuste da técnica estão expostos nas Tabelas 1 e 2; a precisão entre as alíquotas de uma mesma amostra, quer material de referência, quer amostra real, variou de $2 \%$ a $20 \%$ para todos os metais determinados. A exatidão referente ao material de referência certificado foi satisfatória.

Neste artigo em função do objetivo proposto inicialmente, somente será abordado os resultados referentes às frações reativa e pirita.

\section{RESULTADOS}

Os dados obtidos para os metais-traço associados as frações reativa e pirita estão nas Tabelas 3 a 6 . Os valores obtidos sugerem uma distinta especiação nos dois ambientes estudados. Os metais $\mathrm{Fe}, \mathrm{Cr}, \mathrm{Co}, \mathrm{Ni}$ e $\mathrm{Cu}$ variaram de 1,60 a $2,10 \%, 3,50$ a $4,10 \mathrm{mg} / \mathrm{kg}, 5,50$ a $6,80 \mathrm{mg} / \mathrm{kg}, 6.10$ a $7.20 \mathrm{mg} /$ $\mathrm{kg}$ e 9.90 a $12.60 \mathrm{mg} / \mathrm{kg}$ na estação 1 , respectivamente e de 1,70 a $4,10 \%, 6,40$ a $13,40 \mathrm{mg} / \mathrm{kg}, 7,00$ a $15,90 \mathrm{mg} / \mathrm{kg}, 7,80$ a $16,60 \mathrm{mg} / \mathrm{kg}$ e 11,80 a $26,30 \mathrm{mg} / \mathrm{kg}$ na estação 2 , respectivamente. Os metais-traço $\mathrm{Fe}, \mathrm{Cr}, \mathrm{Co}, \mathrm{Ni}$ e $\mathrm{Cu}$, associados a fração reativa nos primeiros $15 \mathrm{~cm}$ de coluna sedimentar, apresentaram uma maior concentração na estação 2 ; entre $0-5 \mathrm{~cm}$, as concentrações dos metais associados a fração reativa na estação 2 foram aproximadamente três vezes superiores as da estação 1; as concentrações de $\mathrm{Fe}, \mathrm{Cr}, \mathrm{Co}, \mathrm{Ni}$ e $\mathrm{Cu}$ na fração reativa, entre $5-15 \mathrm{~cm}$ nas duas estações amostradas, exibiram em sua maioria variações bastante inferiores aquelas mostradas na profundidade $0-5 \mathrm{~cm}$ (Tabelas 3 e 4 ).

Tabela 3. Concentração dos Metais associados a Fração Reativa na Estação 1.

\begin{tabular}{cccccc}
\hline \multicolumn{5}{c}{ Metais na Fração Reativa na Estação 1} \\
\hline Prof. (cm) & $\mathrm{Fe}$ & $\mathrm{Cr}$ & $\mathrm{Co}$ & $\mathrm{Ni}$ & $\mathrm{Cu}$ \\
$(0-2)$ & 1,60 & 3,60 & 5,50 & 6,30 & 9,90 \\
$(2-5)$ & 1,70 & 3,50 & 5,50 & 6,10 & 10,10 \\
$(5-10)$ & 1,90 & 4,10 & 6,80 & 7,20 & 12,60 \\
$(10-15)$ & 2,10 & 3,90 & 5,80 & 6,80 & 11,40 \\
\hline
\end{tabular}

*Fe em $\%$ e $\mathrm{Co}, \mathrm{Ni}, \mathrm{Cu}$ e $\mathrm{Cr}$ em $\mathrm{mg} / \mathrm{kg}$.

Tabela 4. Concentração dos Metais associados a Fração Reativa na Estação 2.

\begin{tabular}{cccccc}
\hline \multicolumn{6}{c}{ Metais na Fração Reativa na Estação 2} \\
\hline Prof. (cm) & $\mathrm{Fe}$ & $\mathrm{Cr}$ & $\mathrm{Co}$ & $\mathrm{Ni}$ & $\mathrm{Cu}$ \\
$(0-2)$ & 4,10 & 13,40 & 15,90 & 16,60 & 26,30 \\
$(2-5)$ & 4,00 & 10,90 & 12,70 & 13,60 & 21,00 \\
$(5-10)$ & 1,80 & 6,60 & 7,80 & 8,20 & 11,70 \\
$(10-15)$ & 1,70 & 6,40 & 7,00 & 7,80 & 11,80 \\
\hline
\end{tabular}

*Fe em \% e $\mathrm{Co}, \mathrm{Ni}, \mathrm{Cu}$ e $\mathrm{Cr}$ em $\mathrm{mg} / \mathrm{kg}$.

As percentagens de $\mathrm{Fe}, \mathrm{Cr}, \mathrm{Co}, \mathrm{Ni}$ e $\mathrm{Cu}$ associados a fração pirita variaram de 0,50 a $3,20 \%, 0,85$ a $1,40 \%, 0,46$ a $7,25 \%, 1,45$ a $2,55 \%$ e 1,18 a $3,55 \%$, na estação 1 respectivamente, e de 0,38 a $0,64 \%, 0,10$ a $0,90 \%, 0,17$ a $0,18 \%, 0,29$ a $1,40 \%$ e 1,20 a $1,50 \%$, na estação 2 respectivamente. Todos os metais estudados, exceto o $\mathrm{Cr}$, exibiram um aumento na associação à pirita com a profundidade na estação 1 , no ambiente com Avicennia o percentual apresentou-se aproximadamente constante ou com uma ausência de acumulação com a profundidade (Tabelas 5-6). Este perfil infere uma acumulação dos minerais sulfídricos na estação 1 e das fases geoquímicas redutíveis na estação 2 . 
Tabela 5. Percentagem dos metais associados a Fração Pirita na Estação 1.

\begin{tabular}{cccccc}
\hline \multicolumn{6}{c}{$\%$ Metais na Fração Reativa na Estação 1} \\
\hline Prof. $(\mathrm{cm})$ & $\mathrm{Fe}$ & $\mathrm{Cr}$ & $\mathrm{Co}$ & $\mathrm{Ni}$ & $\mathrm{Cu}$ \\
$(0-10)$ & 0,50 & 1,36 & 0,46 & 1,45 & 1,18 \\
$(10-20)$ & 1,10 & 0,85 & 2,70 & 2,55 & 2,00 \\
$(20-30)$ & 3,20 & 1,40 & 7,25 & 1,65 & 3,55 \\
\hline
\end{tabular}

*Fe em \% e $\mathrm{Co}, \mathrm{Ni}, \mathrm{Cu}$ e $\mathrm{Cr}$ em $\mathrm{mg} / \mathrm{kg}$.

Tabela 6. Percentagem dos metais associados a Fração Pirita na Estação 2.

\begin{tabular}{cccccc}
\hline \multicolumn{6}{c}{ \% Metais na Fração Reativa na Estação 2} \\
\hline Prof. (cm) & $\mathrm{Fe}$ & $\mathrm{Cr}$ & $\mathrm{Co}$ & $\mathrm{Ni}$ & $\mathrm{Cu}$ \\
$(0-10)$ & 0,56 & 0,90 & 0,18 & 0,90 & 1,30 \\
$(10-20)$ & 0,64 & 0,10 & 0,18 & 1,40 & 1,20 \\
$(20-30)$ & 0,38 & 0,90 & 0,17 & 0,29 & 1,50 \\
\hline
\end{tabular}

*Fe em \% e $\mathrm{Co}, \mathrm{Ni}, \mathrm{Cu}$ e $\mathrm{Cr}$ em $\mathrm{mg} / \mathrm{kg}$.

\section{DISCUSSÃO}

Lacerda e seus colaboradores ${ }^{7}$, estudando a biogeoquímica e distribuição de metais-traço em rizosferas de mangue na Baia de Sepetiba-RJ, encontraram concentrações de sulfetos em solos com Avicennia e Rhizophora iguais a $0.33 \mathrm{mg} / 1$ e $15.6 \mathrm{mg} / \mathrm{l}$ respectivamente. As condições redutoras para o solo com Avicennia foi inferior àquele com Rhizophora, onde encontrou-se potencias de redução iguais a $-63 \mathrm{mV}$ e $-173 \mathrm{mV}$ respectivamente. Os autores citaram que a Avicennia está apta a oxidar sua rizosfera (permitindo assim uma maior concentração de metais-traço na fração reativa), possuindo por conseguinte um maior potencial redox que aquele encontrado em solos com Rhizophora. Segundo os mesmos, os ambientes com predomínio da espécie Avicennia são caracterizados por uma alta variabilidade em suas condições redox e concentração de sulfetos. Evidenciaram este fato por grandes desvios padrões de Eh e [HS ${ }^{-}$, sugerindo constantes mudanças de condições óxidas à anóxicas. A espécie Avicennia germinans seria capaz de conduzir ar pelas raízes, podendo assim crescer em substrato anaeróbico ${ }^{18}$. Até o presente momento não foi cientificamente provado que suficiente volume de ar escaparia das raízes, oxidando o substrato anaeróbico. De qualquer modo parece que este mecanismo seria responsável pela oxidação do $\mathrm{H}_{2} \mathrm{~S}$ na rizosfera ${ }^{15}$.

$\mathrm{O}$ perfil de maior biodisponibilidade de metais foi observado nos primeiros $5 \mathrm{~cm}$ na estação com Avicennia; estes metais estariam associados a óxidos, hidróxidos, carbonatos ou adsorvidos em argilo-minerais e sulfetos metaestáveis, inferindo assim uma menor concentração de minerais sulfídricos nesta região sedimentar. Ao que parece, as soluções sólidas de pirita e sulfetos metálicos, comuns em ambientes sulfídricoanóxico ${ }^{6}$ não se acumulariam na estação 2 , ao contrário do que sugere os dados para a estação 1 .

A formação do sulfeto de ferro 'pirita' em ambiente anóxico sedimentar temperado tem sido bastante estudada ao longo das últimas décadas ${ }^{2,6,8,16,22}$. O mineral pirita é encontrada associada a matéria orgânica, sendo um mineral indicador da diagênese sulfídrica ${ }^{2}$. Estudo feito por Morse ${ }^{13}$ resumiu da seguinte forma os processos formadores de minerais de sulfeto sedimentar ${ }^{2,13,17,20 \text { : }}$

$\mathrm{I}$ - Matéria Orgânica $+\mathrm{SO}_{4}=$ (mediado por bactérias) $\mathrm{H}_{2} \mathrm{~S}$

II - Minerais de ferro $+\mathrm{H}_{2} \mathrm{~S} \cdot \mathrm{AVS}$ (FeS-amorfa, mackinavita e greigita, considerados 'Acid Volatile Sulfides')

III - $\mathrm{H}_{2} \mathrm{~S}+\mathrm{O}_{2}$ (mediado por bactérias) $\mathrm{S}^{0}$

* Parte do $\mathrm{H}_{2} \mathrm{~S}$ proveniente da sulfato-redução é parcialmente oxidado por bactérias à $\mathrm{S}^{0}$.

$\mathrm{IV}-\mathrm{AVS}+\mathrm{S}^{0}$ Pirita $\left(\mathrm{FeS}_{2}\right)$
Estudos feitos em vários ambientes sedimentares no Golfo do México ${ }^{6}$, mostraram que a piritização de metais é um importante processo diagenético ao longo da coluna sedimentar. Em ambientes anóxico-sulfídrico (elevada concentração de $\mathrm{H}_{2} \mathrm{~S}$ e baixa concentração de Fe-reativo) e anóxico-nãosulfídrico (baixa concentração de $\mathrm{H}_{2} \mathrm{~S}$ e elevada concentração de $\mathrm{Fe}$ reativo) existe uma tendência geral de associação com o mineral pirita na coluna sedimentar para $\mathrm{As}, \mathrm{Hg}, \mathrm{Mo}, \mathrm{Co}, \mathrm{Cu}, \mathrm{Ni}$, $\mathrm{Cr}, \mathrm{Pb}, \mathrm{Zn}$; entretanto estes metais são incorporados na pirita em diferentes extensões.

O padrão de associação metal-traço/pirita crescente ao longo da coluna sedimentar, somente foi observado no solo recoberto por Rhizophora. As altas condições sulfídricas neste tipo de rizosfera parecem propiciar a interação e acumulação desta associação. Os metais no solo com Avicennia estariam em menor concentração associados à fração pirita em comparação ao solo com Rhizophora, em função da oxidação dos sulfetos (impossibilitando a formação de um percentual dos sulfetos de ferro metaestáveis, o que afetaria de forma direta a concentração total de pirita no sedimento) na água intersticial e na fase sólida (sulfetos de ferro metaestáveis e pirita); isto explicaria o padrão decrescente (sinônimo da ausência de acumulação) exibido para os metais $\mathrm{Fe}, \mathrm{Co}, \mathrm{Ni}$ e $\mathrm{Cu}$ ao longo do testemunho na estação 2 .

A ausência de acumulação do $\mathrm{Cr}$ ao longo testemunho, concordou com estudos sobre piritização de metais ${ }^{5,6,11}$ (tabelas 3 e 4). Este comportamento seria função da instabilidade dos sulfetos de cromo $^{6,19}$, e uma rápida formação do hidróxido de cromo; esta fase geoquímica competiria com a pirita pelo $\mathrm{Cr}$ em ambiente redutor ${ }^{3,6}$.

Experimento sobre a oxidação de pirita e liberação de $\mathrm{As}, \mathrm{Cu}$, $\mathrm{Hg}$ e Mo em sedimento resuspenso, mostrou que em $88 \%$ dos resultados evidencia-se um percentual médio de oxidação do metal piritizado em torno de $25 \%$. Contudo, a extensão da oxidação do metal foi altamente variável entre um mesmo metal em vários ambientes e entre os metais estudados e uma mesma amostra. Os resultados sugeriram que a incorporação de metais-traço em pirita autigênica é um importante processo diagenético. A maioria das técnicas tradicionais para especiação de metais na fração reativa não incluem a fração pirita. Entretanto, uma porção substancial de metais associados a pirita torna-se reativa se o sedimento for exposto à condições óxidas ${ }^{12}$. O sedimento anóxico é um importante depósito para metais tóxicos e uma matriz controladora de sua biodisponibilidade ${ }^{13}$. Mudanças na redoxclina, dragagens e resuspensões podem tornar estes metais potencialmente biodisponíveis para afetar a cadeia trófica de ambientes aquáticos ${ }^{12}$. $\mathrm{O}$ entendimento dos processos biogeoquímicos ocorridos no ambiente sedimentar propicia uma satisfatória percepção das conseqüências do aporte antrópico nos ecossistemas.

$\mathrm{O}$ estudo da associação de metais à pirita em áreas pouco impactadas ou mesmo sem nenhuma ação antrópica, assim como a costa do Amapá, é de suma importância. Estas áreas são verdadeiros laboratórios naturais para a compreensão dos processos acima citados. Até o presente momento, o estudo de piritização de metais e associação com metais-traço em clima tropical, é praticamente inexistente; os dados obtidos em áreas sem impactação serviriam como linha de base para extrapolações para ambientes contaminados por metais-traço, fornecendo subsídios para um aprofundado estudo de impacto ambiental, controle geral da poluição e critérios de qualidade.

\section{CONCLUSÕES}

As concentrações dos metais-traço $\mathrm{Fe}, \mathrm{Co}$, $\mathrm{Ni}$ e $\mathrm{Cu}$ sugerem que a especiação destes metais nas frações reativa e pirita é diferenciada no sedimento recoberto pelas espécies Avicennia e Rhizophora. A maior concentração dos metais associada à fração reativa no solo com Avicennia, sugere que em função de sua rizosfera possuir um alto potencial oxidante, 
este ambiente propiciaria uma maior biodisponibilidade de metais-traço que aquele recoberto por Rhizophora.

$\mathrm{O}$ padrão de acumulação dos metais-traço na fração pirita ao longo da coluna sedimentar, observado no solo com Rhizophora, sugere que a interação metal/pirita seria mais propícia neste ambiente com condições mais sulfídricas, não influenciando a diagênese de minerais autigênicos de enxofre. A associação de metais-traço a compostos reduzidos de enxofre parece ser diretamente influenciada no sedimento recoberto pela espécie Avicennia.

\section{AGRADECIMENTOS}

Este trabalho foi desenvolvido com bolsa de mestrado CAPES e as análises de elementos traço foram determinadas no CENPES- PETROBRAS, pelas quais agradeço a Dr ${ }^{\mathrm{a}}$ Teresa Cristina da divisão para apoio analítico do ICP-MS. Agradeço ainda ao Dr. Ricardo Santelli pela preciosa ajuda na padronização da técnica analítica.

\section{REFERÊNCIAS}

1. Allison, M. A.; Nittrouer, C. A; Kineke, G. C.; Marine Geology 1995, 125, 303.

2. Berner, R. A.; Am. J. Sci. 1970, 268, 1.

3. Eary, L. E.; Rai, D.; Am. J. Sci. 1989, 289, 180.

4. Harbison, P.; Mar. Pollu. Bull. 1986, 17, 246.

5. Huerta-Diaz, M. D.; Morse, J. W.; Mar. Chem. 1990 , 29, 119.
6. Huerta-Diaz, M. D.; Morse, J. W.; Geochim. Cosmochim. Acta 1992, 56, 2681.

7. Lacerda, L. D.; Carvalho, C. E. V.; Tanizaki, K. F.; Ovalle, A. R. C.; Rezende, C. E.; Biotrópica 1993, 25,252 .

8. Luther III, G. W.; Geochim. Cosmochim. Acta 1982, $146,2665$.

9. Mendes, A. C.; Faria, L. E. C.; III Workshop Ecolab, 1995, Belém, Anais, 127.

10. Mongia, A. D.; Ganeshamurthy, A. N; Agrochim. 1989, 33, 464.

11. Morse, J. W.; Mar. Chem. 1994a, 46, 1.

12. Morse, J. W.; ACS Symposium Series 550, Washington $1994 b, 291$.

13. Morse, J. W.; Ann Arbor Press, Inc. 1995, 187.

14. Naidoo, A. D.; J. S. Afr. Bot. 1980, 46, 293.

15. Nickerson, H. N.; Thibodeau, F. R; Biogeochem. 1985, 1, 183.

16. Raiswell, R.; Plant, J.; Geology 1980, 75, 684.

17. Rickard, D. T.; Stockholm Contrib. Geology. 1969, $20,67$.

18. Scholander, P. F.; L. Van Dam and Scholander, S. I.; Am. J. Botany 1955, 42, 92.

19. Smillie, R. H.; Hunter, K. and Loutit M.; Water Res. 1981, 15,1351

20. Sweeney, R. E \& Kaplan, I. R.; Econ. Geol 1973, 68, 618.

21. Thibodeau, F. R. \& Nickerson, N. H.; Am. J. Sci. 1986, 75, 512.

22. Wang, Q. \& Morse, J. W.; Mar. Chem. 1996, 52, 99. 